\section{Akademie Online - Studentenkurse mit sensationellen Quoten}

Im Januar 2011 hat die Deutsche Röntgengesellschaft mit der Reihe „Akademie Online“ ein neues, digitales Fortbildungsangebot in ihr Programm aufgenommen: Alle 2 Wochen am Dienstagabend ermöglicht es seinen Teilnehmern eine regelmäßige, ortsunabhängige Fortbildung ohne lange Anfahrtswege - ob im Büro, in der Klinik, am heimischen PC, ob allein oder in Gesellschaft, die Fortbildung kommt zum Teilnehmer, nicht umgekehrt. Das neue Format hat innerhalb kürzester Zeit große Akzeptanz bei der Zielgruppe der Radiologen und Weiterbildungsassistenten gefunden; regelmäßig verfolgen zwischen 200 und 300 Teilnehmer die OnlineKurse an ihrem Bildschirm.

Doch nicht nur bei Radiologen, auch bei 100 Medizinstudenten kommt das Angebot sehr gut an: Im Februar 2012 wurde erstmals ein zweiteiliger Online-Kurs für Studenten zur Vorbereitung auf die Prüfung angeboten. Titel: „Keine Angst vor Röntgenbildern im Hammerexamen! “Die Nachfrage nach diesem für Studenten kostenfreien Angebot war schnell so hoch,

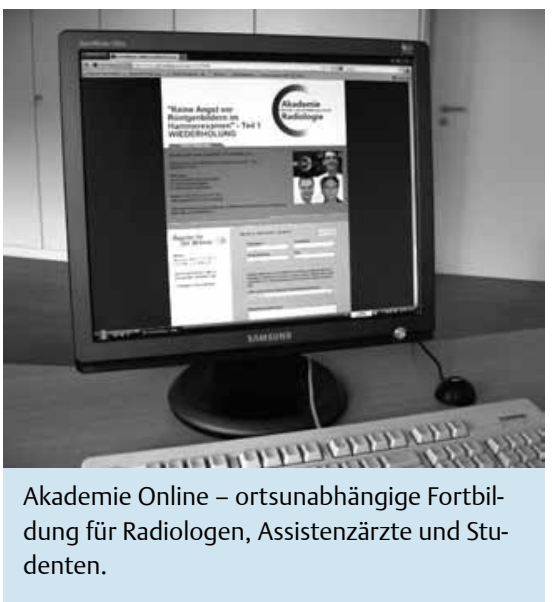

dass kurzfristig 2 Wiederholungstermine im März geschaffen wurden, um jedem Interessenten die Teilnahme zu ermöglichen. Mit jeweils rund 1000 Teilnehmern an allen 4 Terminen wurde die maximale Kapazität des Systems ausgeschöpft sonst wären es wohl noch mehr gewesen.

Die Referenten Prof. Dr. Michael Uder und PD Dr. Rolf Janka aus Erlangen analysierten und diskutierten mit Witz, Charme und natürlich umfassendem Fachwissen zahlreiche Röntgenbilder vergangener Prüfungen und gaben hilfreiche Tipps für die Herangehensweise. Unterstützt wurden Sie dabei von Dr. Florian Mayer, der die Kurse moderierte und „nebenbei“ noch einige 100 Fragen der Teilnehmer beantwortete, die über den Chat gepostet wurden. Diese Form der Prüfungsvorbereitung traf den Nerv der Medizinstudierenden auf den Punkt: Die Feedbacks reichten von „super Seminar“ über „einfach nur Hammer. Toll rübergebracht“ bis hin zu „echt empfehlenswert. Macht Lust auf mehr Radiologie“. Und noch ein weiterer Kommentar zeigt, dass dieses Angebot auch als großer Erfolg für die Nachwuchsarbeit der Fachgesellschaft gewertet werden kann: „Hervorragend! Vielen Dank! Nicht nur für die Examensvorbereitung, sondern einfach die Art und Weise, die Freude am Fach mit seinen Möglichkeiten, die mir die Radio ganz unerwartet so nah gebracht hat, dass ich mich für diesen Facharzt entscheiden werde!“

Die Nachfrage nach mehr Kursen dieser Art ist groß. Besonders oft wünschten sich die Teilnehmer Kurse, in denen es nicht ausschließlich um Prüfungsfragen, sondern auch um die generelle und systematische Herangehensweise an radiologi- sche Bilder geht. Diesem Wunsch entspricht die Deutsche Röntgengesellschaft gern: Im April werden 2 weitere Studentenkurse angeboten, die sich speziell mit dem Röntgenthorax, Knochenröntgen und abdominaler Bildgebung beschäftigen. Und da das Angebot insgesamt auf so positive Resonanz stößt, wird es bei Akademie Online ab sofort wohl in jedem Semester heißen: „Keine Angst vor Röntgenbildern im Hammerexamen“.

Mehr Informationen zu Akademie Online und das Kursprogramm finden Sie unter www.drgakademie.de/site/akademieonline. 